\title{
Analyst
}

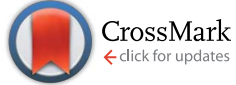

Cite this: Analyst, 2014, 139, 4641

Received 6th May 2014

Accepted 7th July 2014

DOI: $10.1039 / c 4 a n 00811 a$

www.rsc.org/analyst

\section{Determining the chronology of deposition of natural fingermarks and inks on paper using secondary ion mass spectrometry}

\author{
Nicola Attard-Montalto, ${ }^{\text {aa }}$ Jesús J. Ojeda, ${ }^{a}$ Alan Reynolds, ${ }^{a}$ Mahado Ismail, ${ }^{\text {b }}$ \\ Melanie Bailey, ${ }^{b}$ Lisette Doodkorte, ${ }^{c}$ Marcel de Puit ${ }^{c}$ and Benjamin J. Jones ${ }^{d}$
}

\begin{abstract}
This study thoroughly explores the use of time-of-flight secondary ion mass spectrometry (ToF-SIMS) for determining the deposition sequence of fingermarks and ink on a porous paper surface. Our experimental work has demonstrated that mapping selected endogenous components present in natural fingermarks enables the observation of friction ridges on a laser-printed surface, only when a fingerprint is deposited over this layer of ink. Further investigations have shown limited success on ink-jet printing and ballpoint pen inks. 51 blind tests carried out on natural, latent fingermarks on laser-printed surfaces; up to $14^{\text {th }}$ depletion with samples aged for up to 421 days have resulted in a 100\% success rate. Development with ninhydrin was found to affect the fingermark residue through mobilisation of ions, therefore sequencing determination was compromised; whilst iodine fuming and 1,2-indanedione developers did not. This implied that selected development methods affected success in fingermark-ink deposition order determination. These results were further corroborated through inter-laboratory validation studies. The adopted protocol and extensive series of tests have therefore demonstrated the effectiveness and limitations of ToF-SIMS in providing chronological sequencing information of fingermarks on questioned documents; successfully resolving this order of deposition query.
\end{abstract}

\section{Introduction}

Fingerprint evidence is routinely used in forensic investigations and has been a widely accepted form of identification evidence for over 100 years. ${ }^{1}$ Dactyloscopy is, however, a particularly challenging field of forensic science as fingermarks lack consistency, with a composition that depends on the individual, as well as his/her diet, stress levels and grooming regime. Further discrepancies in the appearance of friction ridges are also affected by exerted pressure on application, duration and angle of contact between the finger and a receiving surface. ${ }^{2-5}$ Porous-surfaces in particular affect surface residue deposits because of their inhomogeneous composition and ability to rapidly absorb components into the substrate. ${ }^{6}$ The development treatment necessary to visualise latent prints and analyse ridge detail, which is selected on the basis of factors including substrate and fingermark age, also affects the components within the fingermark. This entire spectrum of factors is further

${ }^{a}$ Experimental Techniques Centre (ETCbrunel), Brunel University, Uxbridge, UB8 $3 P H$, UK. E-mail: nicola.attard-montalto@brunel.ac.uk

${ }^{b}$ Department of Chemistry, University of Surrey, Guildford, Surrey, GU2 7XH, UK ${ }^{c}$ Netherlands Forensic Institute (NFI), Laan van Ypenburg 6, 2697 GB, The Hague, The Netherlands

${ }^{d}$ School of Applied Sciences, University of Huddersfield, Queensgate, Huddersfield, HD1 3DH, UK complicated by the fact that dactyloscopy heavily relies on the skill of the fingerprint examiner to correctly discern the ridge features in the fingermark evidence. ${ }^{7,8}$

When investigating cases of fraud or counterfeiting, besides recovering the fingermark ridge pattern on a handled document, it is necessary to establish whether the fingerprint has been deposited before or after the surface was written or printed over with compromising ink material. This would allow the forensic document investigator to establish the chronology of a fingerprint on a surface and therefore identify whether an individual is actually associated with the ink-related evidence or simply handled a blank sheet of paper. If it was possible to tell whether a document was handled after inked evidence was deposited onto the surface, a forensic investigator would be able to avoid claims of a suspect handling a pre-printed/signed document: if touched after ink deposition, then the suspect/ donor would have handled a pre-printed document. This problem is one of the major challenges in fingermarks associated with document examination, as existing development techniques do not provide any information on chronology or depth of penetration of fingerprints into porous surfaces, making it impossible to determine the order of deposition of fingerprints and inks.

An increasing number of established and emerging analytical characterisation technologies are being implemented to study and retrieve information from fingerprint evidence. These 
include infrared and chromatographic methods, as well as desorption/ionisation-mass spectrometry techniques, which all have particular features that at present allow or are still being explored to investigate fingermarks. Composition, alteration, presence of contaminants, imaging of ridge detail and increasing the level of understanding of interactions experienced between fingermarks and surfaces, environments, development agents and other processes are all aspects being examined for fingermark analysis, which have been described elsewhere. ${ }^{4,9-15}$

In cases of fraud or counterfeiting, the examination of questioned documents and fingermark components on these porous surfaces necessitates an investigative method with good spatial resolution, highly surface-sensitive capabilities and minimal sample preparation owing to the complexity of the chronological information required. A recent study into sequencing of fingermarks and inks by Fieldhouse $e$ t $a l .{ }^{3}$ reports a good degree of efficiency by electrostatic detection (EDSA) on laser printed inks and fingermarks on paper from surface indentations. In our research we explore the application of timeof-flight secondary ion mass spectrometry (ToF-SIMS) as a suitable technique to establish the chronological sequence of printed inks and fingermarks on paper. This instrumental method targets the chemical composition of a sample, providing a combination of elemental and molecular fragment signatures which can be directly linked to individual constituents within a sample complex. It is therefore much more sample-specific and does not purely rely on physical surface indentations. Although other methods such as matrix-assisted laser desorption ionization (MALDI) and desorption electrospray ionization (DESI) are imaging mass spectrometry techniques with proven success in fingermark analysis, ${ }^{\mathbf{1 0}, 13}$ neither has achieved the spatial resolution or surface sensitivity of ToFSIMS. ${ }^{16}$ SIMS uses a finely focussed primary ion beam for the 'soft'-ionization of a surface through desorption of secondary ions. When coupled with a time-of-flight mass spectrometer, this instrumental method allows for rapid mass-spectral analysis over a wide mass range with good mass resolution. It is also capable of producing high resolution chemical images by rastering the ion beam across the sample surface. ${ }^{17}$ ToF-SIMS has excellent lateral resolution capabilities, is particularly surface sensitive, and has recently demonstrated applications in the forensic analysis of fingerprints, ${ }^{18-21}$ papers and paper coatings, ${ }^{22}$ and in determining the deposition order of fingerprints and ball point inks, however with limited success. ${ }^{18,23}$

The present work is a development and expansion of our proof of concept study, ${ }^{24}$ and investigates the use of this technique for chronological determination of inks and natural fingerprints deposited on paper. Rigorous testing has been carried out on a variety of printing papers, overlaid with latent fingerprints acquired from various donors on solid, text and draft laser-printed black and coloured inks. Investigations have explored both latent and developed fingermarks, which were examined in various deposition sequences and at staggered times, with extended ageing periods of over 1 year. Our experimental work has demonstrated that mapping various endogenous components within the fingermark enables the observation of friction ridges from fingermarks on the ink surface when a fingerprint is deposited above a layer of ink. Developers were found to affect the chemical components and resulting ion signal from a fingermark residue to various extents, with certain development methods compromising the ability to determine the order of deposition of these fingerprints on inks. This work therefore also advises the order of which latent fingermarks should be developed on this type of evidence to effectively target the deposition order query.

\section{Experimental}

\subsection{Materials}

Four types of white office $80 \mathrm{~g} \mathrm{~m}^{-2}$ printing paper were used in these experiments. The inks and printing techniques are detailed in Table 1, with a total of 156 known samples and 51 blind-test samples analysed. Both natural and sebaceousloaded samples were initially investigated. Natural samples were, however, considered more representative of the types of marks encountered in field applications and were therefore the principal focus of this study. ${ }^{25}$ Donors were asked not to wash their hands or apply cosmetics for at least 30 minutes prior to fingermark deposition. Fingertips were also rubbed together to improve distribution of sample deposit from both left and right hands. ${ }^{25}$ Fingerprint sampling usually involved acquiring first, second and fifth depletions of all fingers per hand; though in ball point pen tests only first depletions were investigated. Some fingermarks were applied over a layer of ink (fingermark over ink: FOI), while other marks were deposited on plain paper and subsequently printed over with ink (fingermark under ink: FUI). Printing over the fingermarks in FUI samples was executed within 30 minutes of fingermark deposition, unless the fingermark was intentionally left to age before ink deposition. The analysis day in FUI prints therefore generally determined the age of both fingerprint and ink. Ball point pen ink experiments necessitated manual application of ink, with both solid and discrete lines of ball point pen ink marked onto the standard 80 $\mathrm{g} \mathrm{m}^{-2}$ paper. Ink was applied uniformly in all samples, although in solid ink examples this implied that certain areas were inked over more than once. The ink in these ball point pen ink experiments was allowed to dry for four days and 1 month in two separate consecutive studies before fingermarks were deposited onto the inked surface in FOI studies. Fresh and aged ink and fingermark sequences were analysed for all samples. Samples were aged together in a filing cabinet located in a normal office environment, and no particular care taken to avoid inter-sample contact during ageing, packaging or transportation.

\subsection{Development}

A number of samples were developed with ninhydrin and/or iodine for the development trials as detailed in Table 1 . Ninhydrin solution was prepared by mixing $5 \mathrm{~g}$ of ninhydrin (WA UK, SIRCHIE®) with $45 \mathrm{~mL}$ of ethanol, $5 \mathrm{~mL}$ acetic acid and 2 mL ethyl acetate (>99\% purity, Sigma-Aldrich [UK]); to which 1 litre of $3 \mathrm{M}^{\mathrm{TM}}$ Novec HFE 7100 was added. This solution was 
Table 1 An outline of variables and samples tested in the study (does not include inter-lab validation studies)

\begin{tabular}{ll}
\hline Variable & Aspect/number tested \\
\hline Paper & $\begin{array}{l}\text { 4 batches: Lyreco (1) and office depot (3 types). } \\
\text { ALL } 80 \mathrm{~g} \mathrm{~m}^{-2}\end{array}$ \\
Ink type & $\begin{array}{l}\text { 3 laser printers, } 2 \text { photocopiers, } 1 \text { inkjet printer, } 2 \text { black } \\
\text { BIC pens (fine and cristal) }\end{array}$ \\
Ink style & $\begin{array}{l}\text { Printed laser: solid, draft, text } \\
\text { Printed inkjet: solid only }\end{array}$ \\
& $\begin{array}{l}\text { Ballpoint pen: solid, text } \\
\text { Black, cyan, magenta, yellow, red }\end{array}$ \\
$\begin{array}{l}\text { Printed ink } \\
\text { colours }\end{array}$ & $\begin{array}{l}\text { 15 for laser printed inks, 2 (one good, one poor) for } \\
\text { inkjet printing, } 1 \text { donor for ballpoint pens, } 3 \text { for blind }\end{array}$ \\
tests
\end{tabular}

either sprayed or swabbed onto the latent fingermarks with a cotton bud onto the surface, and development was accelerated by placing the samples in an oven with a beaker of water to avoid total desiccation, at $60{ }^{\circ} \mathrm{C}$ for 20 minutes. Documents developed in iodine ( $>99.8 \%$ Sigma-Aldrich) were suspended in an airtight chamber with $1 \mathrm{~g}$ of iodine crystals dispersed in a Petri dish at the base of the chamber. The latent fingermarks were fumed naturally without any additional application of heat or air flow for 45 minutes.

\subsection{Instrumental analysis}

Initial examination of materials was executed using a Zeiss Supra 35VP field emission scanning electron microscope (FEGSEM), coupled with an Oxford Instruments Inca energy dispersive X-ray analyser (EDX). Samples were coated with approximately $11 \mathrm{~nm}$ of gold to increase surface conductivity and enhance contrast. Samples were mounted on aluminium stubs using a carbon-loaded pressure sensitive adhesive. Imaging and elemental composition assessment was conducted under high vacuum at $20 \mathrm{kV}$.

ToF-SIMS was used to individually analyse paper, printed ink and fingermarks deposited on silicon wafer as well as the fingermark-ink FOI/FUI sequencing samples prepared (Table 1). Secondary ion spectra and images were obtained using a Kore Technology Ltd. SurfaceSeer ToF-SIMS spectrometer. Two types of primary ion sources were used: an ${ }^{114} \mathrm{In}^{+}$and a ${ }^{69} \mathrm{Ga}^{+}$FEI liquid metal ion source. The spectrometer was operated in a pulsed, positive mode with $25 \mathrm{kV}$ applied voltage and $1 \mu \mathrm{A}$ current. Secondary ions were analysed in a reflectron time of flight mass spectrometer and detected with a dual microchannel plate assembly. A flood gun with low energy electrons was operated simultaneously to compensate for surface charging in insulating samples. Spatial resolution was $\sim 1 \mu \mathrm{m}$ diameter, and flight times were recorded with a 0.5 ns time-to- digital converter. Mass spectra acquisition time was set at 60 seconds at a magnification of $\times 100$, and acquired spectra $\mathrm{m} / \mathrm{z}$ range was between 0-1300 Daltons (Da). Well-defined cation peaks on these spectra between 10-60 Da were generally used to select mapping regions. $512 \times 512$ pixel chemical maps were acquired over $40-50$ minutes at 20 cycles per pixel for $4 / 5$ frames, with a minimum of three areas scanned per sample. Acquired maps measured $\sim 750 \times 750 \mu \mathrm{m}$, and were processed where necessary to improve contrast using imaging software. The images themselves were visually assessed to establish whether fingermark traces could be observed over an inked area. All ToF-SIMS spectra were calibrated before analysis.

\subsection{Blind tests}

Blind test samples were acquired from one female and two male donors on both solid, draft and text printing in a similar procedure as outlined in 2.1 above. A range of $1^{\text {st }}$ to $14^{\text {th }}$ depletions were prepared, with ink deposited in various ink and fingermark sequences on different days. These were analysed at varied times between 1 to 421 days after fingermark deposition. Samples were cut up and arbitrarily numbered in polyethylene sample bags, and were submitted for ToF-SIMS analysis without providing any additional sample information. Quasi-operational blind tests were also investigated, which replicated an infield scenario much as possible. Here a text, laser-printed document was first developed with iodine (IOD) with fingermark boundaries marked out post development to establish fingerprint location. Samples were then documented, packaged and analysed using ToF-SIMS to determine fingermark chronology (FOI/FUI). Each identified fingerprint was further developed by spraying with ninhydrin (NIN) solution and left in an oven for 45 minutes to visually attest whether a 'standard' developmental procedure was possible after the fingermark sequencing determination protocol.

\subsection{Inter-laboratory validation experiments}

Six known and six unknown, latent and developed samples acquired from 3 donors were analysed in an inter-laboratory validation study executed in collaboration with the University of Surrey and the Netherlands Forensic Institute (NFI). Samples were prepared at the NFI, with fingermarks from thumb, middle and ring fingers deposited $1 \mathrm{~h}$ after donors had washed their hands with soap and water. $80 \mathrm{~g} \mathrm{~m}^{-2}$ paper (Fastprint extra) was used in these experiments, and lines of ink were printed over/ under the fingerprint-paper setup with a standard $1200 \mathrm{dpi}$ laser printer. All blind test samples were developed, with two examples provided for NIN development, and four for 1,2indanedione (IND).

Development was carried out using standard NFI stock solutions. NIN was prepared by mixing $25 \mathrm{~g}$ of ninhydrin (BVDA, Haarlem) and $225 \mathrm{~mL}$ of ethanol; with $10 \mathrm{~mL}$ ethyl acetate and 25 $\mathrm{mL}$ acetic acid ( $>99 \%$ purity, Sigma-Aldrich [Zwijndrecht, the Netherlands]) subsequently added to the stock solution. 1 litre of HFE 7100 (3M St. Paul, USA) was then mixed with $52 \mathrm{~mL}$ of NIN until a homogeneous solution was formed. A similar stock solution of IND was prepared by combining $10 \mathrm{~g}$ of indanedione 
(BVDA, Haarlem) and $60 \mathrm{~mL}$ of ethyl acetate, $10 \mathrm{~mL}$ of acetic acid and $900 \mathrm{~mL}$ HFE 7100. A working solution was prepared by mixing $8 \mathrm{~mL} \mathrm{ZnCl}_{2}$ (>99\% purity, Sigma-Aldrich, prepared with ethanol, ethyl acetate and HFE 7100) with $100 \mathrm{~mL}$ indanedione. Both NIN and IND development was executed via immersion, with samples dipped in solutions until wet. These were then air dried for a few minutes and heated for 10 minutes at $80{ }^{\circ} \mathrm{C}$ and 20 minutes at $100^{\circ} \mathrm{C}$ for NIN- and IND-treated samples respectively.

ToF-SIMS investigations for the inter-laboratory experiments were carried out at the University of Surrey 68-72 days after the samples were developed, and no particular care was taken in the packaging or transportation of the samples. Analysis was executed on an IONTOF GmbH (Münster, Germany) ToF-SIMS 5 spectrometer, employing a $25 \mathrm{keV}^{3{ }^{3+}}$ primary ion beam delivering $0.35 \mathrm{pA}$ of current. Images were acquired at $128 \times$ 128 resolution in the MacroRaster mode over a $5 \times 5 \mathrm{~mm}$ area. Image data were acquired using 256 cycles per pixel point with 1 scan per pixel and a cycle time of $100 \mu \mathrm{s}$.

\section{Results and discussion}

\subsection{Laser printed ink and photocopying}

Laser printers and photocopiers engage electrostatic lifting of toner particles onto a charged, rotating drum. When the drum comes into contact with a sheet of paper, an image is printed onto the paper surface through the application of direct contact (pressure) and heat, which fuses the ink to the paper. SEM images of laser, draft-printed and photocopied paper all demonstrated this fused toner, which formed a discrete layer of ink with several surface irregularities and voids that completely covered the underlying interwoven mass of paper fibres. In draft-printed ink at $300 \mathrm{dpi}$, ink covered less than half the paper surface in a checkerboard pattern at a relatively small scale, yet still masked the paper fibres (Fig. 1a and b). EDX analysis of these toner-based inks showed their fused-silica, organic-based nature. Additional elemental colorants, opacifiers and/or additives were also identified, with iron consistently detected in black inks, copper and titanium in cyan, and titanium with traces of sulphur in both magenta and yellow printed toners. Sodium and calcium peaks were present in all EDX spectra, identified as well-known paper additives sodium chloride and calcium carbonate. ${ }^{\mathbf{2 4 2 2}}$

ToF-SIMS data of samples of fingermarks over and under laser-printed ink (FOI/FUI) acquired in this study corroborated
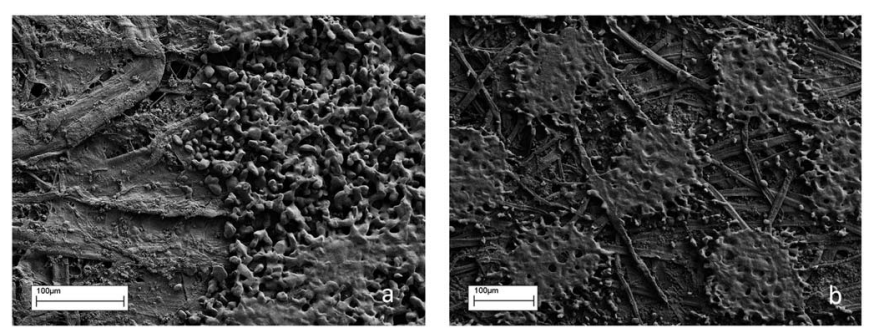

Fig. 1 Secondary electron images of photocopied ink (a) at the paperink interface and (b) an image of draft printed ink. the preliminary results published in our proof of concept study. ${ }^{24}$ Ions in the lower mass range were consistently more prominent in all mass spectra owing to analyte composition and ionising properties of the primary ion beam. ${ }^{22}$ Mapping of these ions presented clear secondary ion maps with improved ion contrast and material visibility. These lower $\mathrm{m} / \mathrm{z}$ cations were therefore identified as the most useful species to differentiate between fingermark, paper and ink constituents. Mapped ions included the paper derivatives calcium $\left(\mathrm{Ca}^{2+}\right)$ and to a lesser extent quaternary nitrogen $\left(\mathrm{C}_{3} \mathrm{H}_{8} \mathrm{~N}^{+}\right)$at 40.078 and 58.102 Daltons respectively. ${ }^{22}$ Silicon $\left(\mathrm{Si}^{+}\right)$and $\mathrm{SiOH}^{+}$at $\mathrm{m} / z 28.086$ and 45.093 Da were identified as the embedding silicon components in laser-printed inks. The organic nature of the samples implied that various hydrocarbon fragments at, $m / z$ 15.034, 27.045, 29.061, 41.071 and 55.098 Da, for example, were identified in all materials being examined (paper, ink, fingerprint). The most prominent hydrocarbon peak generally appeared at $\mathrm{C}_{3} \mathrm{H}_{5}{ }^{+}$ (41.071 Da). Relative heights of each component to the surface also affected constituent ion counts. This was as a result of the surface sensitivity of the instrument and subsequent limitations in height resolution over a specific area, with larger distances increasing the time taken for secondary ions to reach the time of flight mass analyser, resulting in contrast differences and identification of laser-printed ink and paper boundaries (Fig. 2).

Sodium $\left(\mathrm{Na}^{+}\right)$and potassium $\left(\mathrm{K}^{+}\right)$secondary ions of 22.990 Da and 39.098 Da, consistently corresponded to the fingermark deposit. $\mathrm{Na}^{+}$ions typically dominated as a base peak in fingermark spectra, though these salts sometimes appeared as accretions in maps around pore areas from certain donors. Replicated samples analysed from donors indicated that certain individuals consistently deposited poorer fingermarks, though certain other deposits appeared to be affected by cold weather. This was demonstrated by analysing average ion counts and image contrast in SIMS maps. When a mass spectrum was acquired at a non-pore $/ \mathrm{Na}^{+}$accretion area in poorer donors, this was reflected through comparatively lower peak heights in the mass spectra at these particular areas. Sodium ions also predominated in all examined paper substrates, especially in the Office Depot paper batches. The similarity between $\mathrm{Na}^{+}$and $\mathrm{C}_{x} \mathrm{H}_{y}{ }^{+}$mass fragments in paper and fingerprint component fractions implied that locating a fingermark deposited on paper from these ions was difficult; whether or not the paper was subsequently printed over with ink (FUI). Occasionally, the sodium ion counts in the fingermark were notably higher than those in the paper, implying that ridges could be identified, but these concentrations varied widely between paper and fingerprint donors, as well as in depletion, day and time of deposition.

Sodium ions were additionally observed as base peaks in mass spectra acquired from cyan and yellow toners, as well as being fairly prominent in magenta mass spectra (Fig. 3). $\mathrm{K}^{+}$was also abundant in all coloured laser inks, with ion counts in secondary ion maps approximating those in FOI fingermarks, therefore making it difficult to discern ridges in potassium maps. The $\mathrm{Na}^{+}$peak and respective ion counts on mapping were consistently higher in samples where the fingerprint was deposited over laser printed inks, even in poor donor samples. 

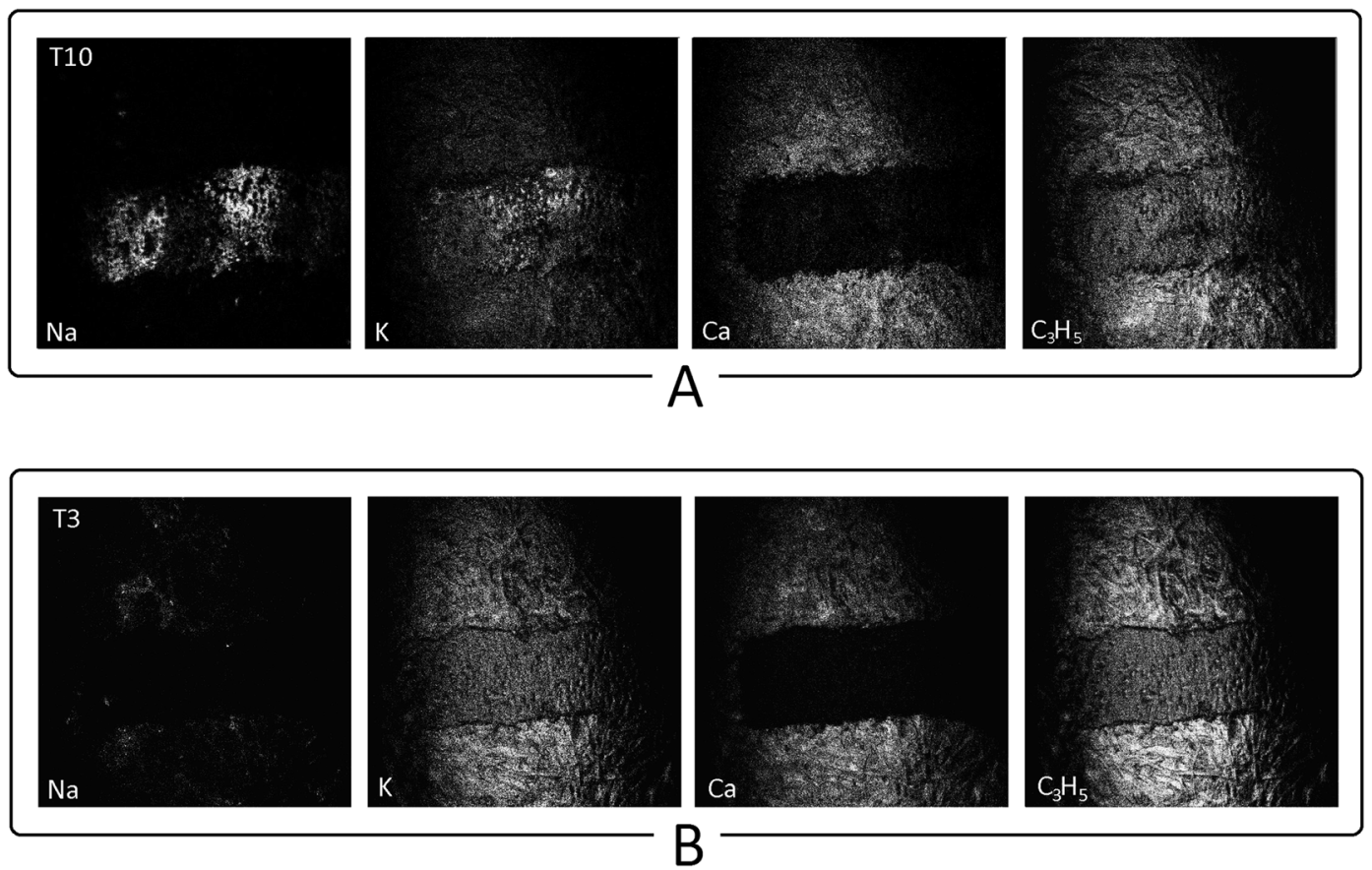

Fig. 2 Examples of positive secondary ion maps of $\mathrm{Na}, \mathrm{K}, \mathrm{Ca}$ and $\mathrm{C}_{3} \mathrm{H}_{5}$ ions from a $5^{\text {th }}$ depletion fingermark over (T10 series $-\mathrm{A}$ ) and a $1^{\text {st }}$ depletion under (T3 - B) ink, both acquired from the same donor and aged for 170 days (image size $\sim 750 \times 750 \mu \mathrm{m}$ ).

This implied that determining the fingermark chronology over these inks was still possible (Fig. 3).

Increasing the donor pool, experimenting with different printing styles (photocopies, text and draft printing), and testing aged inks and fingerprints were used to establish relative sensitivity and potential limitations of this ToF-SIMS technique. Following a broad, varied sample study of fingermarks on/under laser-printed ink, factors appearing to affect interpretation were generally a combination of fingerprint donor and printed ink characteristics. Compromising factors

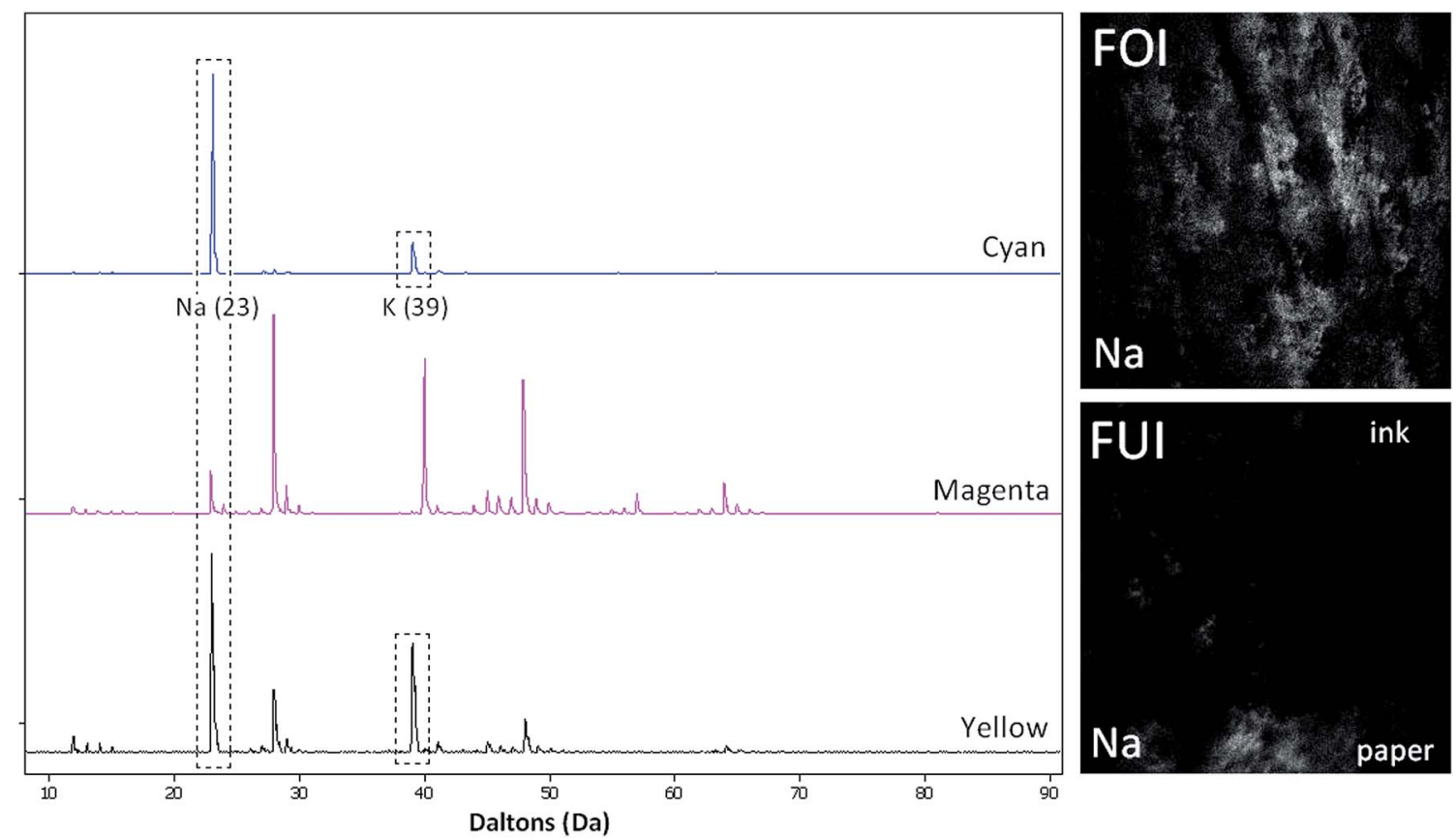

Fig. 3 Stacked mass spectra of cyan, magenta and yellow laser printed inks, showing the elevated sodium and potassium peaks in the toners, with maps comparing $\mathrm{Na}^{+}$maps of an FOI and FUI sample over cyan ink. 
included lower ion counts resulting from decreased quantities of natural secretions in collected fingermarks, either as a result of them being significant depletions in a depletion series, and/ or because the deposit was acquired from a poor donor, and/or on a cold day. Furthermore, as the paper provided similar ion fractions to the distinguishing secondary ions in the fingermarks, identifying ridges in FOI samples was found to depend on the amount of ink deposited on the paper surface, with text and draft-printed ink making data analysis more difficult. Fingerprint age, ink age, as well as ink colour did not appear to particularly affect interpretation, as was demonstrated through the successful identification of FOI/FUI sequences where fingermarks and inks had been aged for over a year. This indicated that ion mobility through a stratum of ink with time did not compromise FOI/FUI determination in this type of printed ink. Nevertheless, aged fingermarks on draft-printed $300 \mathrm{dpi}$ inks were more difficult to identify, especially after the samples had been aged for a substantial time period (S60 Fig. 8). Furthermore, although fingermarks over coloured laser-printed inks could not be distinguished in respective $\mathrm{K}^{+}$maps; it was always possible to ascertain an FOI fingermark from the elevated ion counts in $\mathrm{Na}^{+}$maps.

\subsection{Inkjet printing}

Inkjet printing is an alternative deposition technique used for liquid phase inks that consist of an organic colorant solute, dissolved or dispersed in a solvent. A fixed quantity of ink is ejected from the printer nozzle, where it spreads under momentum and surface tension along a surface (Fig. 6). Studies have shown that drop spread is dependant on the viscosity of the polymer, the height of the nozzle, as well as the structure of the receiving surface. ${ }^{26-28}$

This wetting-penetration effect was reflected in results obtained, where SEM-EDX analysis generally did not allow for the discernment of printed areas on paper as there was no particular difference in height, composition, or in the appearance of paper fibres, presence of voids and crystallinity of filler aggregates within the paper (Fig. 4). Interface edges of coloured ink and paper in effect required light marking with a ball point pen to identify printed and non-printed paper. A variation in colour was only observed in black ink-jet printed area; though EDX results did not identify any particular difference between this black ink and paper components. Compositional analysis in all samples merely indicated that calcium was dominant in
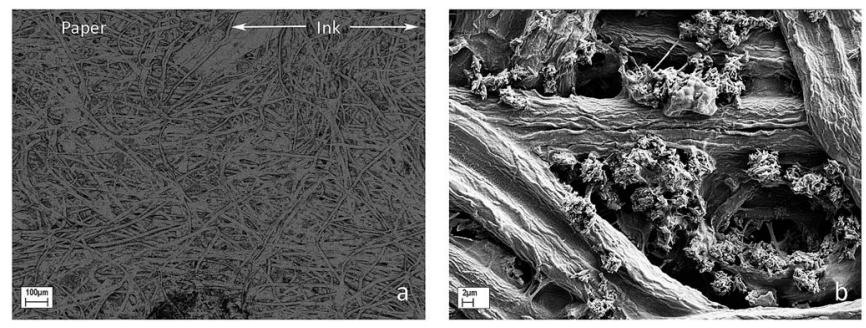

Fig. 4 SEM images of (a) yellow ink-jet ink on paper (BSE, interface) and (b) filler aggregates in an inked area of the paper. the paper, whether or not it was inked over, and that the inks were organic. This organic nature was confirmed by ToF-SIMS analysis, with most ink fragments observed characteristically denoting long-chain hydrocarbon fractions, with similar major mass spectral peaks obtained from cyan, magenta and yellow inks.

Sequencing trials of fingermarks over and under printed inkjet inks were executed on fresh ('wet') and older ('dry') samples. A slight difference in the strata height and composition of the paper and inked areas was observed in the maps, allowing for identification of paper-ink interfaces, and of printed areas. Initial ion maps analysed from fresh FOI/FUI samples with freshly printed inks gave poor results, mainly owing to outgassing effects experienced on UHV conditions, which resulted in poor ion counts. Chemical images designated swollen printed paper fibres, indicative of the absorption of these 'wet' inks into the paper cellular structure, which expanded as a result of this ink application. Fingermark ridges were near-invisible in this fresh-sequencing setup, although an increased amount of salts were observed in a few FOI samples; typically those obtained from the thumb and index fingers from a better donor. This was not a consistent result and therefore implied the methodology was unsuitable for ridge identification on freshly printed ink-jet paper surfaces.

Observation of aged samples showed that printed paper fibres had dried and returned to their original shape and structure. Fibre edges in the black printed areas were somewhat less defined than those in the coloured samples, suggesting that this black ink was deposited as a thicker layer. This could also account for the backscatter greyscale difference between the paper and black ink observed in the SEM images (Fig. 4). Although ion counts in aged samples were improved, sequencing determination of FOI and FUI fingermarks was still inconclusive. In black inkjet-fingermark examples, no ridges were observed in the FOI/FUI samples examined, making it impossible to ascertain whether a fingermark was at all present in a mapped location. In the coloured ink samples, ridges were observed in the majority of the sodium ion maps in the inkedover areas of the paper. These ridges, however, were not limited to FOI sequences but were also observed in the FUI samples as in Fig. 5c.

Fig. 6 schematically demonstrates the differences between the two printing techniques. Toners in laser-jet printing effectively fuse onto a surface in a 'dry' process, forming the discrete layer of ink observed in SEM images and SIMS maps. Inkjet ink is conversely deposited in solution, therefore penetrates into the paper, colouring the paper fibres and drying level to the surface (Fig. 6a). When a fingerprint is deposited onto a printed document, a mixture of fingermark components are brought into direct contact with the document surface. The isolating stratum of fused Si-based toner in laser-jet ink is assumed to decrease the porosity of the paper surface, implying that the fingermark components rest on top of this relatively impermeable barrier' formed by the ink layer. The altered porosity also explains why migration of components is not observed after samples are aged for significant periods of time, implying that fingerprint components are not allowed to interact with the 

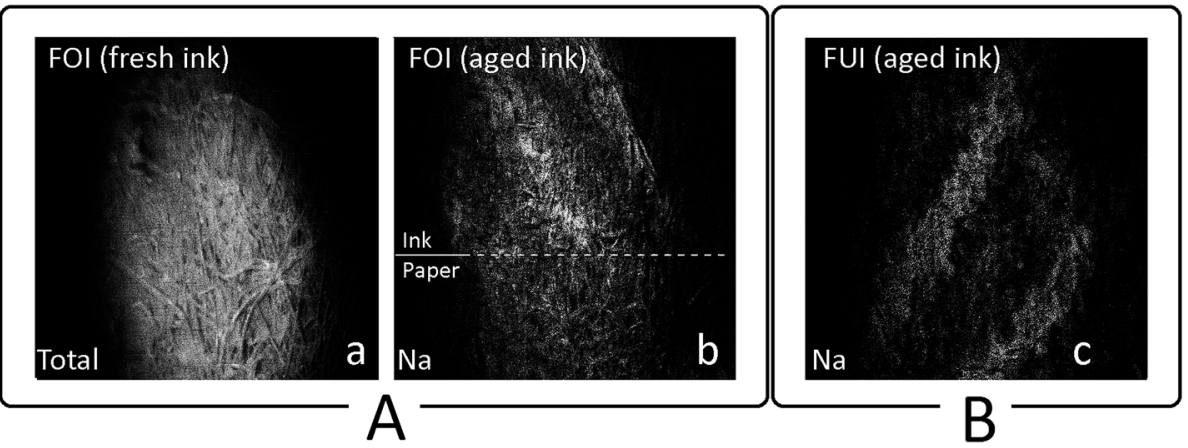

Fig. 5 (a) SIMS map demonstrating swollen fibres in freshly deposited black ink. The other two maps show observable ridge detail in the $\mathrm{Na}^{+}$ maps from FOI (A) and FUI (B) samples on magenta ink. Both samples are thumb prints: (a) is an interface example and (b) is an ink only example.

porous paper surface and therefore remain on the ink surface and are identifiable by ToF-SIMS. Conversely, inkjet ink does not form this isolating layer but is deposited by the printer as a 'wet' solution. This is demonstrated in the visible paper fibres of the inked-over areas, even after the ink has dried (Fig. 4 and 5).

\subsection{Ballpoint pens}

Mass spectroscopic techniques including ToF-SIMS have previously been used to explore the composition of ballpoint pen inks, their drying and ageing characteristics; as well as the deposition order of fingermarks over/under these inks on paper. ${ }^{11,23,29-31}$ Work carried out in this study corroborated published results, with ToF-SIMS compositional analysis indicating that inks from the separate BIC pens examined in this study were both similar, organic-based complexes. Basic crystal violet dye $\left[\mathrm{C}^{+}(\mathrm{Me})_{6}\right]$ was also observed at $372.526 \mathrm{Da}$, representing the characteristic colorant in standard black and blue ball point pens. ${ }^{11}$ Non-quantitative results on older ink samples in this study also suggested the loss of methyl groups via the breakdown of this basic violet dye owing to the apparent reduction in height of $\mathrm{m} / \mathrm{z} 372.526 \mathrm{Da}$ and the appearance of additional peak clusters at $\mathrm{m} / \mathrm{z} 358.499$ and $344.473 \mathrm{Da}$ $\left[\mathrm{C}^{+}(\mathrm{Me})_{5} \mathrm{H}_{1}\right.$ and $\mathrm{C}^{+}(\mathrm{Me})_{4} \mathrm{H}_{2}$.

Paper fibres were still visible through the ink layer, especially when a single line of ink was drawn on the surface. Comparatively low sodium ion levels and the presence of basic violet peaks in the ink nonetheless sufficiently allowed for discrimination between paper and ink, and for the visualisation of a paper-ink interface. This also facilitated friction ridge discernment, generally from elevated $\mathrm{Na}^{+}$levels in FOI samples. Ridge detail was easier to ascertain on 'solid' inks, rather than those over inked lines, owing to the elevated ink coverage. Paper fibres were, however, still mostly visible with this ink type.

Our FOI/FUI order of deposition experiments also supported previous deposition order experiments with ball point pen inks. ${ }^{23}$ The investigations carried out in this study however looked at different mass fragments: $\mathrm{Na}^{+}$in preference to the ink peak intensities at $m / z 372$ and $358 \mathrm{Da}$. This was because these ions were endogenous to the fingerprints and were also the focus of our study, as well as being the base peaks in the

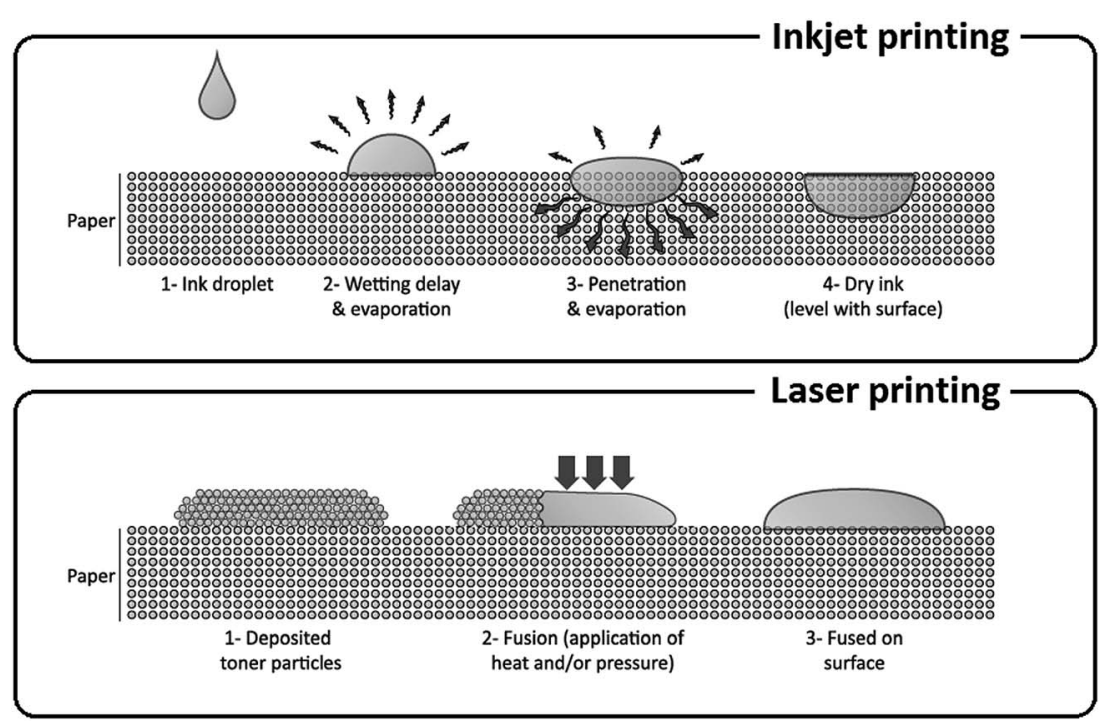

Fig. 6 Diagram showing difference (a) between 'wet' ink-jet printing and (b) and the 'dry' process of laser printing. 

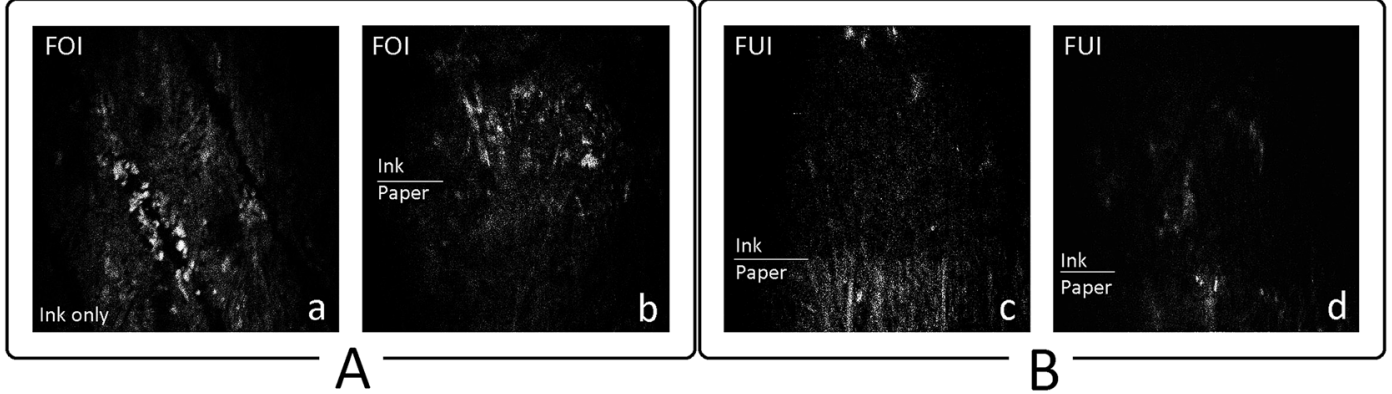

Fig. $7 \mathrm{Na}^{+}$maps from first depletions of a thumb print (a and c), an index print (b) and a ring finger FUI print (d). (a) is a fresh fingermark on a 4 day aged ink, (b) is a 2 day old fingermark on a 6 day old ink (c) is a 2 day old ink and fingermark (d) is a 10 day old ink and fingermark. Samples b-d are interface samples.

majority of the mass spectra. The smaller field of view provided by our instrument also meant that smaller, ink-dominant areas could be mapped. Results consistently presented elevated $\mathrm{Na}^{+}$ levels in FOI samples, but were found to be similarly inconclusive in FUI samples, with some $\mathrm{Na}^{+}$accretions observed in FUI examples. Variation in ion levels was attributed to the variability observed between samples, with influencing factors including quantity of material in the fingermark residue itself as well as ink thickness in the manually drawn ink lines on a paper surface. This in turn affected the overall thickness of the isolating layer of ink and its ability to mask sodium ions in the paper. Improved results were acquired with thumb and index prints, where ridge detail was consistently observed in FOI examples (Fig. 7a and b). Line-drawn examples were more difficult to interpret. The protocol, therefore, although providing an improved result to inkjet FOI/FUI sequencing, still gave an inconclusive result.

\subsection{Blind tests}

A comprehensive blind test series that looked at 51 latent fingermark samples deposited over/under laser-printed ink resulted in a success rate of $100 \%$. This corroborated our previous work and showed that this ToF-SIMS methodology is suitable for determining the chronology of a fingermark over/under laser-printed ink. Results tallied with observations given for known samples, where $\mathrm{Na}^{+}$ions were visibly identifiable over the ink, only in FOI samples. These ions followed fingerprint ridge detail and demonstrated up to third level features. Observations were generally corroborated in $\mathrm{K}^{+}$maps, with examples of approximately 6 month old $5^{\text {th }}$ depletion FOI sample and a $1^{\text {st }}$ depletion FUI over/under text provided in Fig. 2. A number of examples were provided to test those variables identified as limiting factors of our methodology, as identified previously (3.1). Aged samples, quantity (coverage) of ink on the surface as well as depletion prints were analysed, with the oldest, poorest coverage and $14^{\text {th }}$ depletion examples displayed in Fig. 8. S50 shows that ridge detail was still visible in even $\left(4^{\text {th }}\right)$ depletion samples left to age for over one year, only when the fingermark overlies the ink, as can be seen when compared to the FUI equivalent in S21. Furthermore, mobile ions did not appear to migrate through laser-printed ink from the paper, therefore quantity of sodium ions on an ink surface or a fingermark were not affected with time and interpretation was not compromised, even after ageing for over 421 days (S21). The $14^{\text {th }}$ depletion over text (T117) was the maximum depletion sample tested, which still consistently showed salt accretions from pore areas over the lettered ink. Aged, depletion fingermarks over/under draft-printed ink looked at a combination of factors negatively affecting fingermark quality for interpretation. Draft-printed ink in particular reduced the quantity of isolating layer of ink on a much smaller scale. S60, a $3^{\text {rd }}$ depletion aged for just over a year, displayed mere traces of salts over the inked-over areas of this 300 dpi ink. $\mathrm{Na}^{+}$ions nevertheless visibly corresponded to equivalent $\mathrm{K}^{+}$accretions that followed faint ridge detail over ink in respective maps, therefore strongly suggesting a FOI. Amounts visible, however, were minimal, therefore interpretation, especially when purely based on visual examination, could be queried and attributed to possible contamination.

\subsection{Development}

Ninhydrin (NIN) development appeared to compromise our ability to discern fingermark components over ink in FOI samples with the certainty that was observed in the latent fingerprint equivalents. Contact application of NIN with a cotton swab also appeared to have a diluting, and a seemingly smearing effect on the surface residue, making maps difficult to interpret, ridges difficult to identify and results generally inconclusive. A good donor provided better ion contrast in FOI samples with visibly elevated sodium ion peaks over ink, though several areas needed to be mapped as images were less definitive, especially when text samples and/or fifth depletions over ink were examined. Furthermore, in certain FUIs $\mathrm{Na}^{+}$ions appeared to migrate over the ink, especially around ink-paper boundaries (Fig. 9). This was assumed to be a result of mobilisation of salt ions through contact application of the solventbased (wet) developer with a cotton swab over the surface. The presence of $\mathrm{Ca}^{2+}$ ions, which were characteristically limited to paper in non-developed samples, over the ink in both FOI and FUI maps, further supported this material migration/smearing hypothesis, besides designating possible contamination effects. Samples developed through non-contact spray application of 

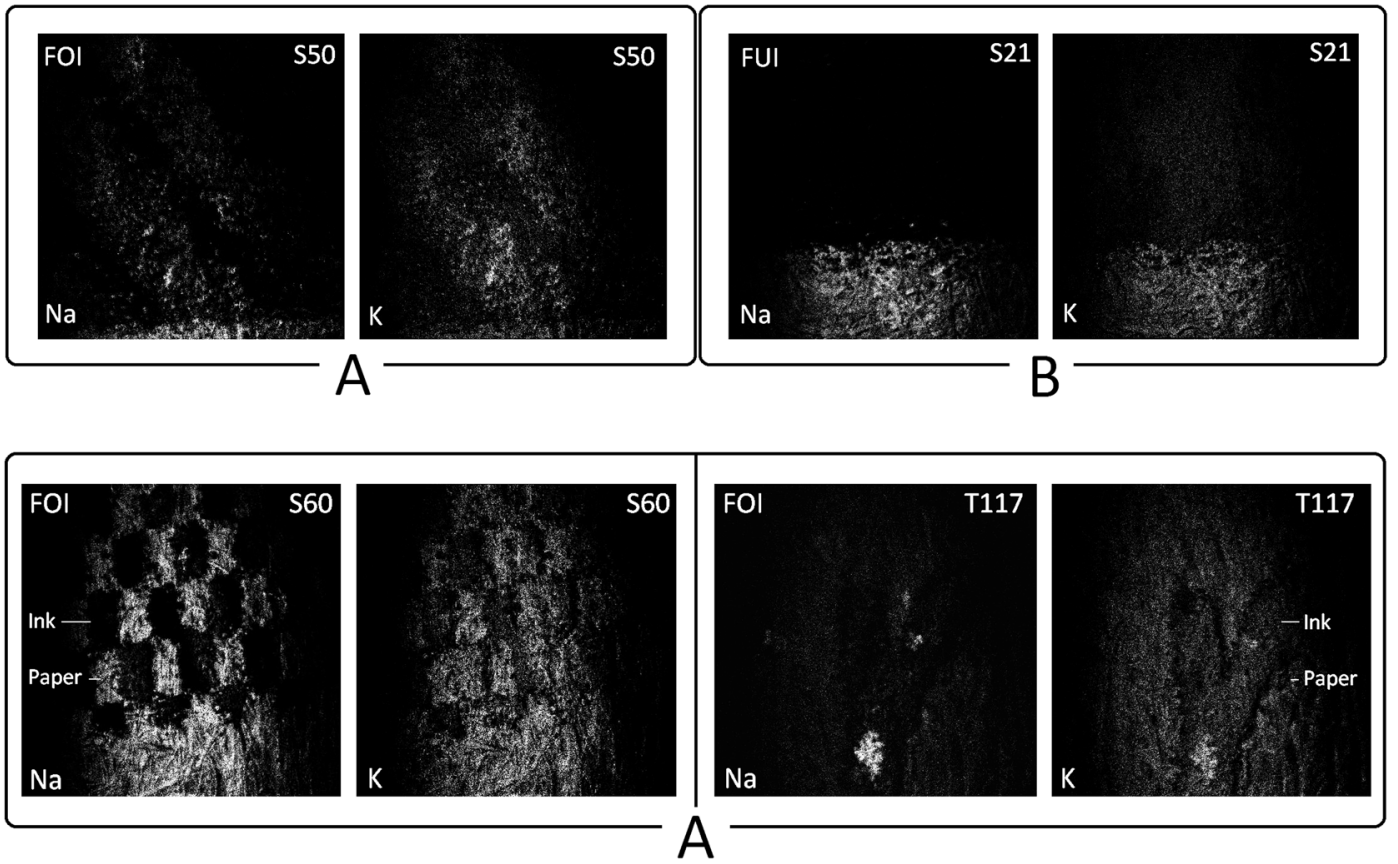

Fig. 8 Examples of $\mathrm{Na}^{+}$and $\mathrm{K}^{+}$secondary ion maps from $\mathrm{FOI}(\mathrm{A})$ and $\mathrm{FUI}(\mathrm{B})$ blind tests. $\mathrm{S} 50$ is a $4^{\text {th }}$ depletion FOI from a female donor and $\mathrm{S} 21$ is a first depletion FUI from a male donor. Both were left to age for 421 days before analysis. 560 is a draft-printed $3^{\text {rd }}$ depletion FOI, analysed 385 days after deposition and T117 is a $14^{\text {th }}$ depletion FOI.
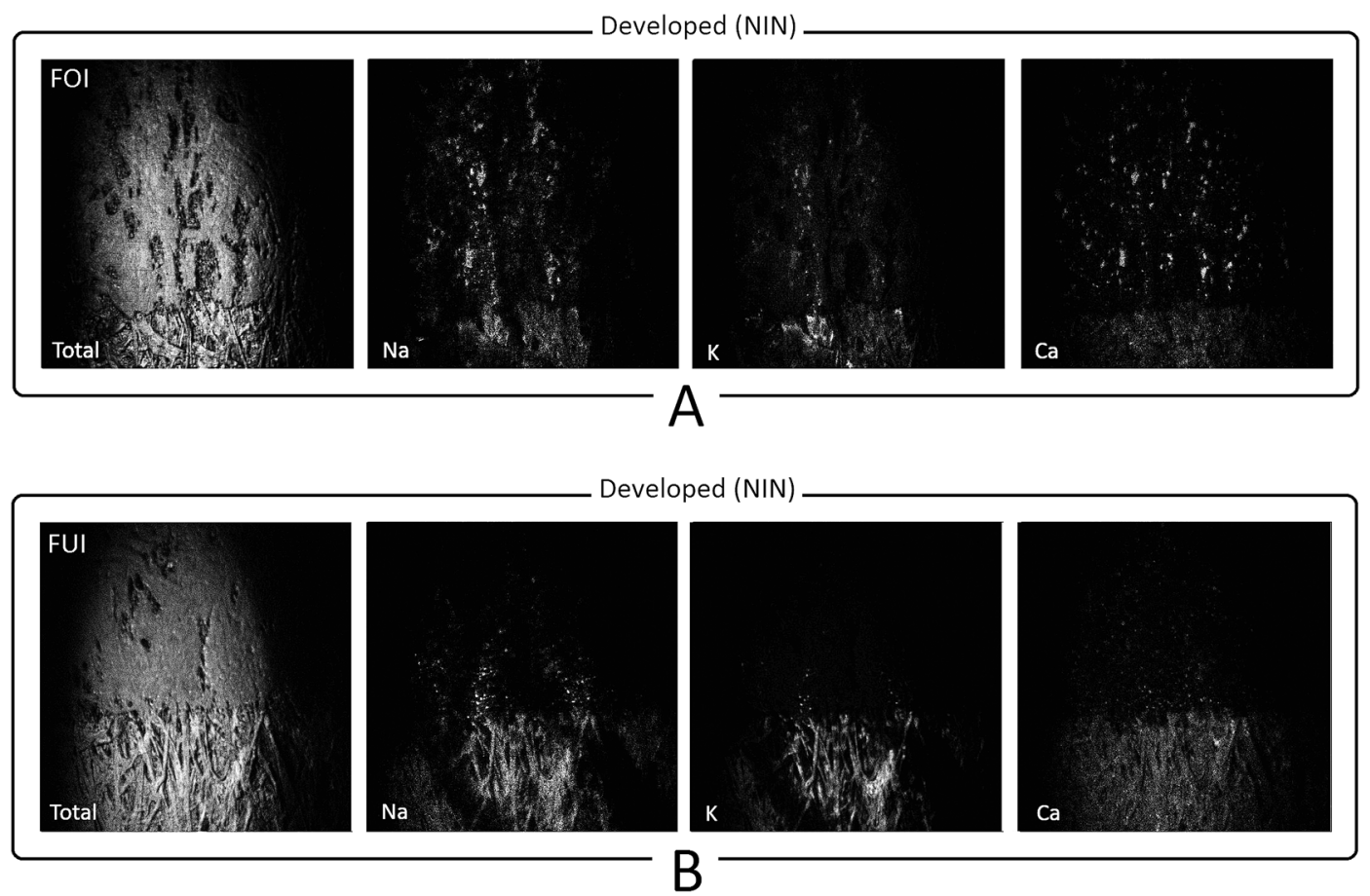

Fig. 9 Examples of FOI (A) and FUI (B) positive SIMS maps from NIN-developed samples.

NIN still had a diluting effect, though secondary ion maps had improved contrast levels when compared to swab application. $\mathrm{Na}^{+}$ions, however, did not follow friction ridge detail and $\mathrm{Ca}^{2+}$ accretions were still present over the ink in both FOI and FUI samples, albeit in lesser quantities. Furthermore, faint $\mathrm{Na}^{+}$ accumulations indicative of salt migration/smearing were again observed over laser printed ink in certain FUI examples. This subsequently compromised data interpretation as poor FOIs acquired from either a depletion sample and/or a poor donor produced maps with similar levels of ion contrast. NIN 
development therefore significantly affected the ability to discriminate between a fingermark overlying or underlying the ink.

Iodine (IOD) was explored as a non-contact alternative, solvent-free developer. Its vapours are assumed to be physically absorbed into the fatty acids in the fingermarks in a transient, reversible process. ${ }^{32}$ This made it particularly desirable for developing the fingermarks in this study owing to a potentially less impinging and detrimental effect on fingermark constituents when compared to NIN development.

Initial maps generated from samples developed with IOD presented strong $\mathrm{Na}^{+}$and $\mathrm{K}^{+}$secondary ion signals over the ink in FOI samples. This observation was consistent, even with $5^{\text {th }}$ depletions. Results were corroborated through a six-donor sample study that looked at split prints, where one half of print was left undeveloped and the other half fumed with iodine. The simultaneous ToF-SIMS analysis and mapping of these samples allowed for effective comparison of data, where mass spectra were both stacked and overlaid for comparative purposes, and maps compared as shown in Fig. 10. There were no prominent differences in relative peak heights between non-fumed and fumed fingerprints. Minor peak-height variations observed were assumed to be a result of inter-sample differences, owing to the presence of more ridges/material/salts in one half of the print than in the other, within a particular area, despite comparisons being made from splits of the same fingermark.

Fig. 10 shows the total, $\mathrm{Na}^{+}, \mathrm{K}^{+}$and $\mathrm{Ca}^{2+}$ secondary ion maps, and compares undeveloped $v s$. fumed, FOI and FUI maps; from a known, relatively poor donor. The total and $\mathrm{Ca}^{2+}$ maps indicate the location of the printed text, whereas $\mathrm{Na}^{+}$and $\mathrm{K}^{+}$maps
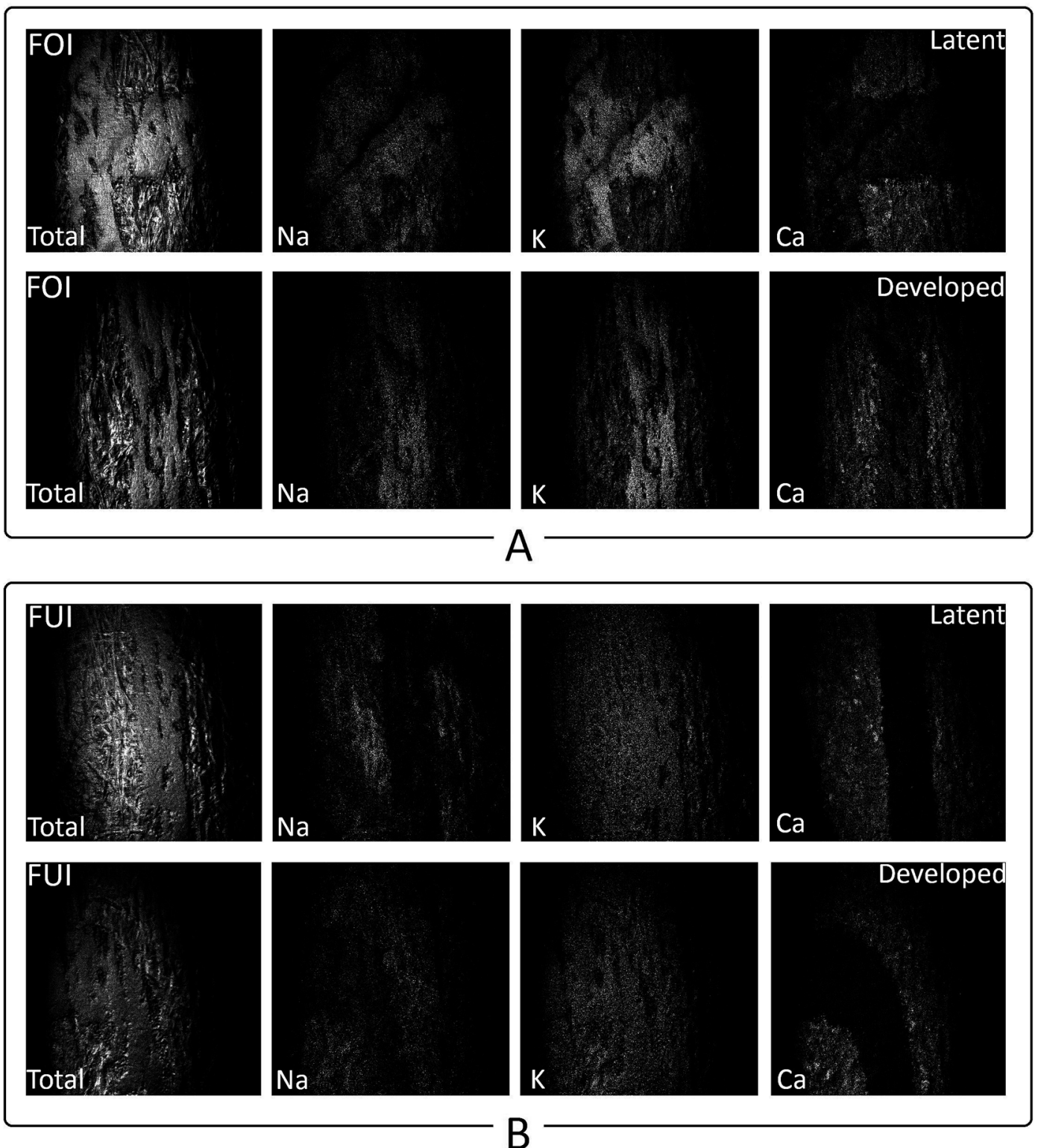

Fig. 10 Total, $\mathrm{Na}^{+}, \mathrm{K}^{+}$and $\mathrm{Ca}^{2+}$ secondary ion maps of split FOI (A) and FUI (B) prints acquired from the same donor: one half of print was left undeveloped (latent) and the other half fumed with iodine. 
show the difference in abundance of these ions over the ink in the FOI $v s$. FUI samples. FOI results clearly show that the fingermark overlies the ink, whether the sample was IOD fumed or left undeveloped. The latent FUI sample also presents an elevated $\mathrm{Na}^{+}$signature along fingermark ridges on the paper beneath the ink, indicating that in this particular example, it was also possible to identify the FUI on the paper. These observations were corroborated in maps acquired from the other 5 donors.

The final blind sample test was executed to test the viability of the experimental protocol through analysis of a laser-printed document with fingermarks possibly deposited, before or after the ink present. This therefore made the test quasi-operational, it being the scenario expected with samples acquired from forensic investigation cases. Five out of the six fingermarks deposited on the laser-printed text from this quasi-operational study were located through iodine development, and all samples were correctly identified as FOIs following ToF-SIMS analysis. Fingerprint samples were further developed with NIN, and developed fingermarks showed excellent contrast and ridge detail, therefore indicating that the methodology for FOI/FUI determination (IOD fuming and ToF-SIMS analysis) did not affect further (amino acid) development for ridge-pattern identification.

\subsection{Inter-laboratory validation}

Imaging latent fingermarks deposited over laser-printed ink on paper in the IONTOF GmbH ToF-SIMS in the Surface Analysis Laboratory at the University of Surrey provided similar results to those described above (3.1). An abundance of hydrocarbon fragments as well as $\mathrm{Na}^{+}$and $\mathrm{K}^{+}$ions at $m / z 22.990$ and 39.098 respectively were observed corresponding to ridge detail in secondary ion maps in FOI samples. The heavier $\mathrm{Bi}^{3+}$ primary ion beam also resulted in increased levels of data from fingermark ion fragments in the higher mass range of the spectrum, such as those at $\mathrm{m} / \mathrm{z} 65.973,87.971$ and 284.326 Da (Fig. 11 and 12). The IONTOF GmbH ToF-SIMS was additionally able to scan a wider area of the sample, with a maximum field of view of $6000 \times 6000 \mu^{2}$ obtained. This produced images with increased amounts of ridge detail, but also implied that the $\mathrm{Na}^{+}$ ions could not be used to distinguish between FOI/FUI samples because of their abundance in the paper substrate which consequently affected data analysis. Furthermore, hydrocarbon data present in the fingermark was also not as identifiable over the ink lines (Fig. 11 and 12). $\mathrm{K}^{+}$ions were, however, visibly elevated in ridge detail in FOI maps, and were therefore consistently used to distinguish the order of deposition of fingermarks on/under ink lines with this instrument.

Sample development by NIN immersion again compromised the ability to identify whether the sample was an FOI/FUI, with $\mathrm{K}^{+}$and other ion fragments appearing over the ink, even in FUI samples. The 'NIN effect' described previously was corroborated by the results obtained in this inter-laboratory validation study, from both known and blind-test samples analysed (Fig. 11).

1,2-Indanedione (IND) was preferentially selected owing to its frequency of use in the NFI as a developer for fingermarks on paper. Development with this reagent gave excellent results, although one sample demonstrated that the quality of donor residue affected interpretation confidence. $\mathrm{K}^{+}$ion maps $(\mathrm{m} / \mathrm{z}$ $39.098 \mathrm{Da}$ ) generally demonstrated clear ridge detail over the

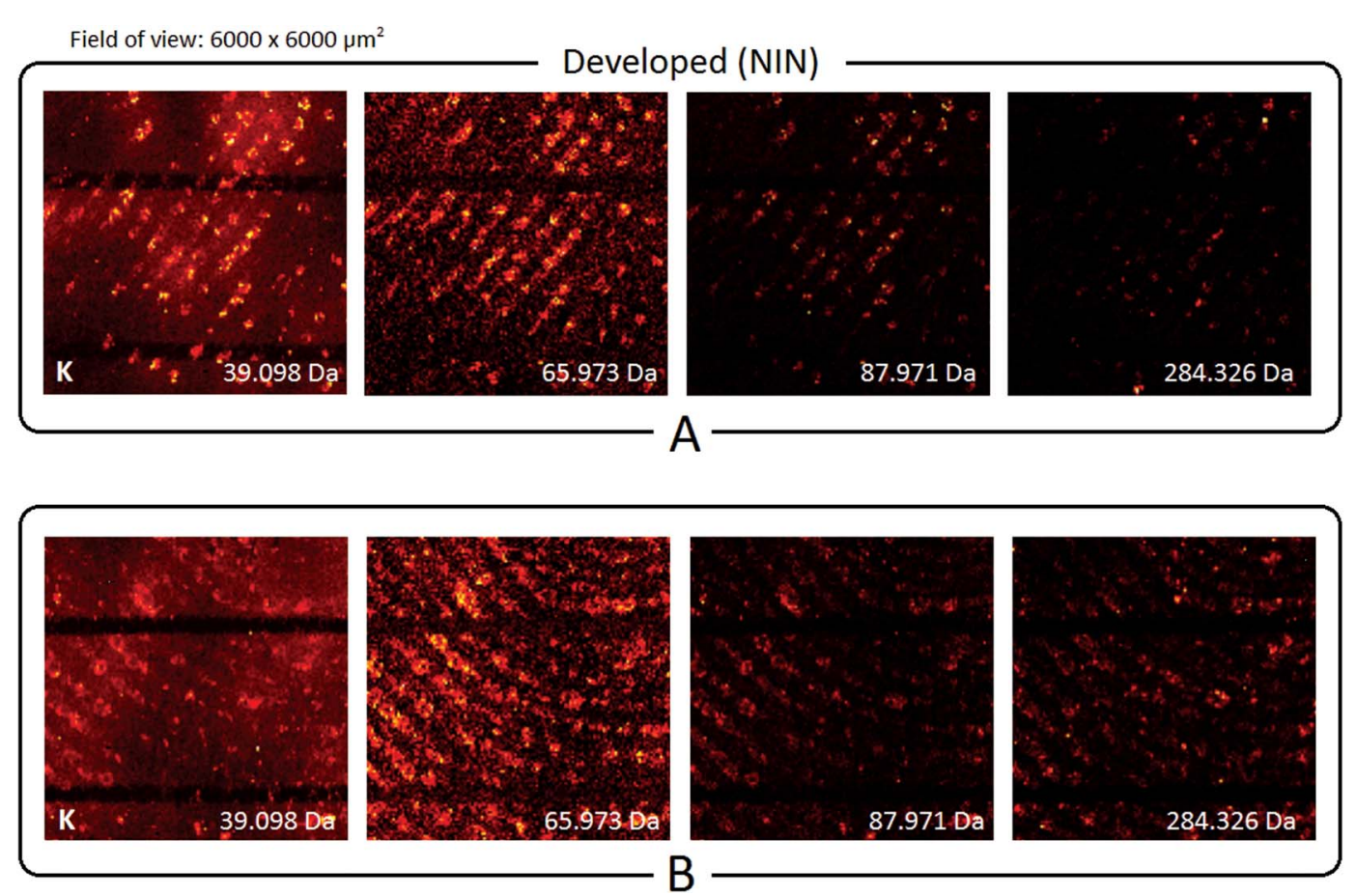

Fig. 11 Positive ion SIMS maps of (A) a fingermark deposited over the ink and (B) ink lines deposited over a fingermark, post-development with NIN. 

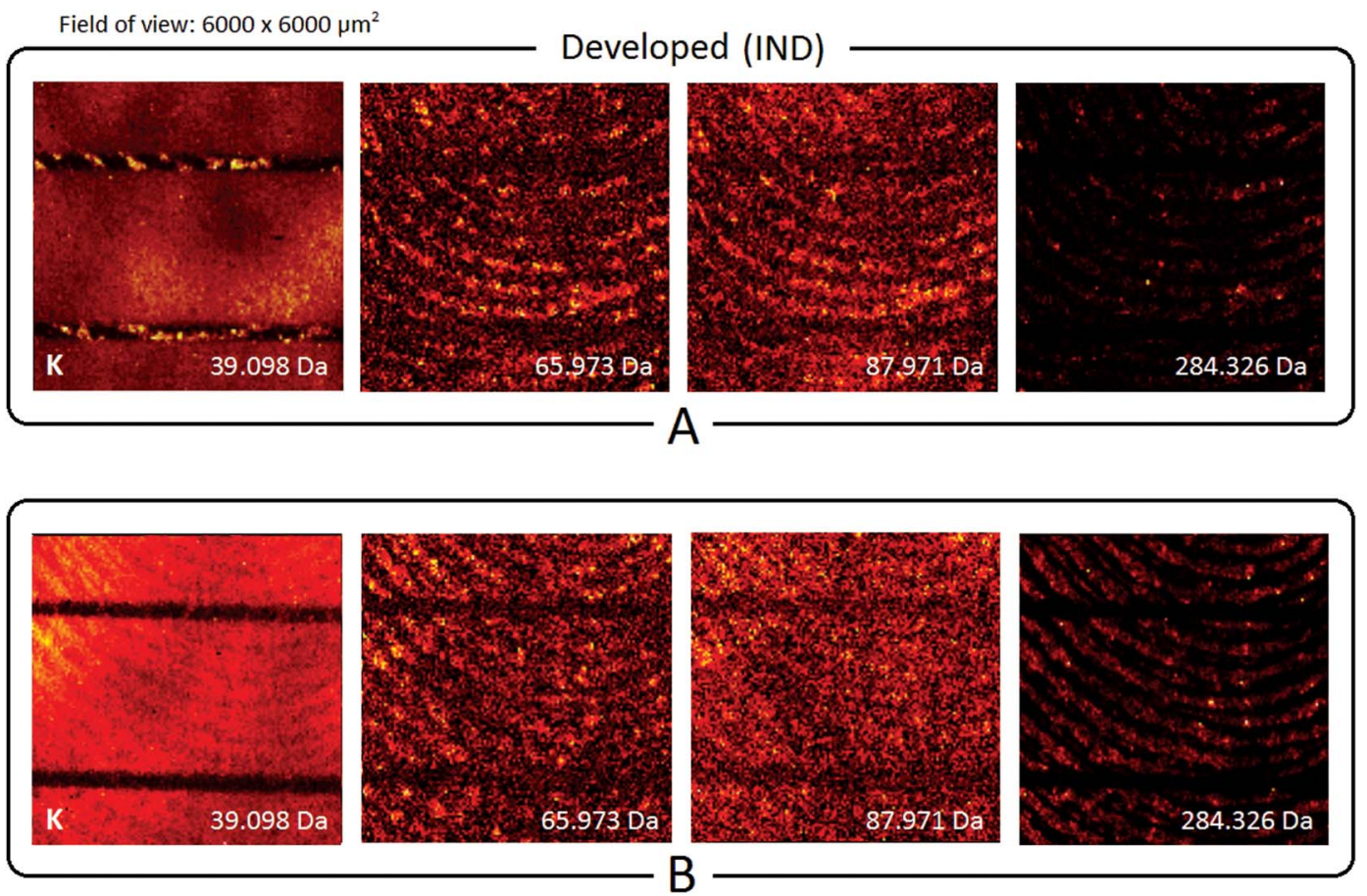

Fig. 12 Positive ion images of (A) a FOI and (B) FUI, post-development with 1,2-indanedione.

ink only when the fingermark had been deposited over this inked layer. Potassium ions could not be identified on the paper substrate using this ToF-SIMS, or over the inked line when an FUI sample was examined. These observations were corroborated in the blind tests studies as demonstrated in the example provided in Fig. 12 below.

\section{Conclusions}

This paper demonstrates that this ToF-SIMS protocol can be used to identify the chronological sequence of natural fingermarks over laser printed ink on paper, and is therefore suitable to verify whether a suspect's fingermark is actually associated with this type of printed evidence. Selected endogenous markers within the fingermark, particularly $\mathrm{Na}^{+}$and $\mathrm{K}^{+}$ions in secondary ion maps jointly show elevated ion counts and ridge detail over the laser-printed ink, only when a fingermark overlies an ink line. Our extensive sampling trials and blind tests indicate that several factors affect quality and subsequent interpretation of results, with reliability generally depending on (i) $\mathrm{Na}^{+}$and $\mathrm{K}^{+}$ion count of in a fingermark, (ii) amount of ink present on the surface and (iii) ink type. A depletion print acquired from a poor donor, for example, denotes a lower ion count, making FOI/FUI sequencing identification more complicated, but still possible, as long as the fingermark was deposited on laser-printed ink. Text and draft laser-printed ink reduced the quantity of isolating layer of ink, implying a smaller area for FOI/FUI determination; again making interpretation more difficult but possible. Ink colour and sample age did not appear to affect acquired data on laser-printed ink. Successful fingermark chronology determination with ball point pen ink depended on the type and amount of ball point pen ink deposited on the surface, with thinly applied and solvent-based inks drying level to the paper surface and therefore compromising sequencing determination. This was because successful identification of $\mathrm{Na}^{+}$and $\mathrm{K}^{+}$markers in a fingermark over the ink necessitated a relatively impermeable ink barrier over the paper, which restricted ion mobility and therefore enabled ridge identification. ToF-SIMS was therefore unsuitable for the identification of the deposition order of a fingerprint on inkjet ink. Development with ninhydrin to locate the latent fingermark prior to ToF-SIMS analysis also affected the reliability of the technique, but the successful results obtained with iodine fuming and indanedione indicated their operational effectiveness, as was demonstrated in the quasi-operational blind test studies and the inter-laboratory validation assessment.

\section{Acknowledgements}

This project is supported by funding from The Leverhulme Trust (RPG-138). The study design, analysis, interpretation and reporting were conducted by the authors with no involvement from The Trust. This research has been approved by Brunel University Research Ethics Committee. The authors would also like to acknowledge the assistance of Ashley Howkins and Nita Verma with the SEM analysis, and thank all their fingerprint donors.

\section{References}

1 M. Page, J. Taylor and M. Blenkin, Uniqueness in the forensic identification sciences-fact or fiction?, Forensic Sci. Int., 2011, 206, 12. 
2 J. Almog, et al., Fingerprints' third dimension: the depth and shape of fingerprints penetration into paper-cross section examination by fluorescence microscopy, J. Forensic Sci., 2004, 49(5), 1.

3 S. J. Fieldhouse, N. Kalantzis and A. W. G. Platt, Determination of the sequence of latent fingermarks and writing or printing on white office paper, Forensic Sci. Int., 2011, 206, 155.

4 A. Hemmila, J. McGill and D. Ritter, Fourier transform infrared reflectance spectra of latent fingerprints: A biometric gauge for the age of an individual, J. Forensic Sci., 2008, 53(2), 369.

5 N. Jones, et al., The influence of polymer type, print donor and age on the quality of fingerprints developed on plastic substrates using vacuum metal deposition, Forensic Sci. Int., 2001, 124, 167.

6 J. S. Dalton, et al., Investigation into the distribution of ink components throughout printed coated paper Part 2: Utilising XPS and SIMS, Colloids Surf., A, 2002, 205, 199.

7 R. A. Hicklin, J. Buscaglia and M. A. Roberts, Assessing the clarity of friction ridge impressions, Forensic Sci. Int., 2013, 226, 106.

8 B. T. Ulery, et al., Accuracy and reliability of forensic latent fingerprint decisions, Proc. Natl. Acad. Sci. U. S. A., Early Ed., 2011, 1.

9 M. J. Bailey, et al., Chemical Characterization of Latent Fingerprints by Matrix-Assisted Laser Desorption Ionization, Time-of-Flight Secondary Ion Mass Spectrometry, Mega Electron Volt Secondary Mass Spectrometry, Gas Chromatography/Mass Spectrometry, Xray Photoelectron Spectroscopy, and Attenuated Total Reflection Fourier Transform Infrared Spectroscopic Imaging: An Intercomparison, Anal. Chem., 2012, 84, 8514.

$10 \mathrm{~S}$. Francese, et al., Beyond the ridge pattern: multiinformative analysis of latent fingermarks by MALDI mass spectrometry, Analyst, 2013, 138(15), 4215.

11 D. M. Grim, J. Siegel and J. Allison, Evaluation of Desorption/ Ionization Mass Spectrometric Methods in the Forensic Applications of the Analysis of Inks on Paper, $J$ Forensic Sci, 2001, 46(6), 1411.

12 D. R. Ifa, et al., Latent fingerprint chemical imaging by mass spectrometry, Science, 2008, 321, 805.

13 M. Morelato, et al., Forensic applications of desorption electrospray ionisation mass spectrometry (DESI-MS), Forensic Sci. Int., 2013, 226, 10.

14 C. Ricci and S. G. Kazarian, Collection and Detection of Latent Fingermarks Contaminated with Cosmetics on Nonporous and Porous Surfaces, Surf. Interface Anal., 2010, 42, 386.
15 C. Ricci, et al., Chemical Imaging of Latent Fingerprint Residues, Appl. Spectrosc., 2007, 61, 514.

16 M. K. Passarelli and N. Winograd, Lipid imaging with timeof-flight secondary ion mass spectrometry (ToF-SIMS), Biochim. Biophys. Acta, 2011, 1811, 976.

17 P. Fardim and B. Holmbom, ToF-SIMS imaging: a valuable chemical microscopy technique for paper and paper coatings, Appl. Surf. Sci., 2004, 249, 393.

18 M. J. Bailey, et al., Depth profiling of fingerprint and ink signals by SIMS and MeV SIMS, Nucl. Instrum. Methods Phys. Res., 2010, 268, 1929.

19 S. J. Hinder and J. F. Watts, SIMS fingerprint analysis on organic substrates, Surf. Interface Anal., 2010, 42, 826.

20 M. I. Szynkowska, et al., ToF-SIMS application in the visualization and analysis of fingerprints after contact with amphetamine drugs, Forensic Sci. Int., 2009, 184, e24.

21 M. I. Szynkowska, et al., Detection of exogenous contaminants of fingerprints using ToF-SIMS, Surf. Interface Anal., 2010, 42, 393.

22 P. Fardim, et al., Extractives on fiber surfaces investigated by XPS, ToF-SIMS and AFM, Colloids Surf., A, 2005, 255, 91.

23 N. J. Bright, et al., Determination of the Deposition Order of Overlapping Latent Fingerprints and Inks using Secondary Ion Mass Spectrometry, Anal. Chem., 2012, 84, 4083.

24 N. Attard-Montalto, J. J. Ojeda and B. J. Jones, Determining the order of deposition of natural latent fingerprints and laser printed ink using chemical mapping with secondary ion mass spectrometry, Sci. Justice, 2013, 53, 2.

25 V. G. Sears, et al., A methodology for finger mark research, Sci. Justice, 2012, 52, 145.

26 P. J. Heard, et al., Visualisation of the distribution of ink components in printed coated paper using focused ion beam techniques, Colloids Surf., A, 2004, 244, 67.

27 M. Singh, et al., Inkjet Printing-Process and Its Applications, Adv. Mater., 2010, 22, 673.

28 D. B. Wallace, et al., Photo-realistic ink jet printing through dynamic spot size control, in IS\&T's $11^{\text {th }}$ Int. Congress in NonImpact Printing Technologies, 1995.

$29 \mathrm{~J}$. Coumbaros, et al., Application of time of flight secondary ion mass spectrometry to the in situ analysis of ballpoint pen inks on paper, Forensic Sci. Int., 2009, 193, 42.

$30 \mathrm{~J}$. Siegel, et al., The use of laser desorption/ionization mass spectrometry in the analysis of inks in questioned documents, Talanta, 2005, 67, 425.

31 C. Weyermann, et al., A GC/MS study of the drying of ballpoint pen ink on paper, Forensic Sci. Int., 2007, 168, 119.

32 C. Champod, et al., Fingerprints and other ridge skin impressions, International forensic science and investigation series, CRC Press LLC, USA, 2004. 